\title{
Applying Diversity Management Concepts to Improve the Minority Educational Pipeline
}

\author{
Joy Oguntebi ${ }^{\dagger}$ \\ Saunders College of Business, Rochester Institute of Technology, Rochester, NY 14623, \\ e-mail: joguntebi@saunders.rit.edu \\ Maria Shcherbakova \\ Saunders College of Business, Rochester Institute of Technology, Rochester, NY 14623, \\ e-mail:mxs2339@rit.edu \\ Lynn P. Wooten \\ Stephen M. Ross School of Business, University of Michigan, Ann Arbor, MI, 48109, \\ e-mail:lpwooten@umich.edu
}

\begin{abstract}
The objective of this conceptual article is to investigate existing diversity management paradigms and extend their implications toward the goal of increasing minority representation in management education. We suggest that the existing learning-andeffectiveness diversity management paradigm (Thomas \& Ely, 1996, Harvard Business Review, 74(5), 79-90), which integrates contributions from diverse individuals, can be utilized in addressing the underrepresented minority shortage at each progressive stage of the education pipeline, beginning with the high school level. We propose and discuss a complex set of solutions that highlight the students' educational experience, motivation, awareness, and social environment. These solutions target minority underrepresentation with the goal of ultimately increasing the proportion of minorities in the $\mathrm{PhD}$ pool and subsequently within the management professoriate.
\end{abstract}

Subject Areas: Diversity Management, Educational Experience, Educational Pipeline, Human Resource Management, Management Education, Minority Education, Minority Representation.

\section{INTRODUCTION}

Researchers have been investigating diversity and inclusion efforts within the workforce, which have largely been motivated by the expanding diverse composition of traditional ethnic groups. In the United States, for instance, it has been observed that the minority population is growing more rapidly than the non-minority population (Gagnon, Totten, \& Morgan, 2007). However, in some areas, including post-secondary education and the professoriate, minorities are proportionally underrepresented. According to estimates, historically underrepresented minorities

\footnotetext{
${ }^{\dagger}$ Corresponding author.
} 
(URM; African-Americans, Hispanic-Americans, and Native Americans) comprise less than $7 \%$ of all U.S. faculty in business and management education (AACSB , 2010), at a time when these minority groups comprise nearly a third of the nation's population (US Census, 2009).

Today, children comprise the largest proportion of multicultural Americans, which illustrates the trend toward diversity-expansion within the U.S. population. Ethnic diversity is being recognized as one of the United States' greatest assets, providing a richness and strength to its economy and culture. Consequently, we have observed that a larger number of organizations are instituting diversity initiatives to effectively execute their objectives and compete advantageously (Harvey, 1999; Kuczynski, 1999). Thus, we explore the success of such successful diverse management strategies and seek to extend implications from these findings to the academic sector. Specifically, we suggest approaches for improving the diversity pipeline in management education. We approach this topic from a sector-level talent management perspective; that is, we conceptualize diversity as a talent management strategy that creates value by recruiting, developing, leveraging, and retaining diverse individuals.

Inclusion and diversity management, which considers the recruitment, development, and retention of URM, are ongoing developments that organizations in a variety of sectors continuously seek to employ. In this conceptual article we will focus on the management education sector, analyzing the importance of diversity within the professoriate by reviewing the diversity trends of other sectors and investigating existing diversity management paradigms that have been developed from both theoretical and practical perspectives. We will also explore the structural reasons for this underrepresentation of minority faculty in management education and suggest structured solutions to addressing this disparity. Both our theoretical perspectives and insights from practice focus on the institutional opportunities and constraints for improving a minority educational pipeline, and the value proposition of investments in diverse individuals.

Today, multiple organizations across different industries are evaluating the effectiveness of their diversity management programs. As Daniels (2001) noted, "in more than $75 \%$ of Fortune 100 companies that have instituted diversity initiatives, the management of diversity has become an important business imperative" (cited by Roberson, 2006, p. 212). Initially, the determinant trends that pushed companies and organizations toward thinking about increasing diversity within the workforce were demographics and social responsibility factors. According to Gagnon et al. (2007), as the U.S population is growing, from 281 million in 2000 to a projected 432 million in 2060, the country's demographics are changing. Racial and ethnic minority groups will contribute more to the population growth than the nonHispanic White population. Ultimately, the projection is that by 2060 , minorities will comprise $53.2 \%$ of the population, making them the dominant population and, consequently, the workforce of paramount importance (U.S. Census Bureau). Moreover, from an ethical and social responsibility perspective, an argument can be made for the necessity to address the underrepresentation of minorities within organizations, and corporations have acknowledged that doing so is imperative for the success of the organization (Barak, 2005). 
In the higher educational context, institutions and their stakeholders have recognized that having diverse communities that are inclusive of URM results in a multicultural learning environment that provides an array of opportunities (Maruyama, Moreno, Gudeman, \& Marin, 2000). For instance, professors view diversity as a resource that enhances learning through exposure, shared experiences, and stimulating conversations (Maruyama et al., 2000). For all students, diverse and inclusive learning environments expand their personal, social, and moral growth capacity and enable universities to achieve their mission. Moreover, a diverse and inclusive learning environment prepares college students for the realities of a pluralistic and global society (Friedman, 2005).

\section{THEORETICAL BACKGROUND}

Significant changes in the ethnic composition of the growing population as well as globalization are among the most significant trends dictating the new rules of managing human resources, not only in business but in all types of organizations. These trends signify a transformation in the collective approach to diversifying the labor force. An extensive review of the literature on the impact of diversity on team and organization performance revealed two dichotomous opinions. One perspective categorizes diversity as a limitation, viewing it as a liability or hindrance to a company's performance. For instance, Bell, Connerley, and Cocchiara (2009) point out that diversity is usually perceived negatively and that the media contribute to the development of such an attitude. This "negative" view is based on theories which emphasize the drawbacks of diversity over its advantages. For instance, the cultural distance hypothesis, introduced by Grubb and Ollendick (1986), assumes that differences in behavior and other processes can be a reason for different levels of performance between URM and their non-URM counterparts. Relationally, the cultural familiarity theory holds that firms are less likely to invest in culturally distant countries, and that they show poorer performance when they do (Lee, Shenkar, \& Li, 2008). Furthermore, Stahl, Mäkelä, Zander, and Maznevski (2010) point to numerous examples of the "disease" orientation in perceiving diversity, noting the significant issues that such an orientation can influence, including team cohesiveness and social integration. Findings from the disease orientation of diversity management highlight the challenges of managing diversity and the potential for negative outcomes, such as increases in conflict and inhibition of decision-making (Stevens, Plaut \& Sanchez-Burks, 2008). These examples that illustrate the limiting perception of diversity are not foreign to the academic environment.

Similarly, other research studies propose that it is difficult to quantify the advantages of diversity management because the business case has not been clearly defined, and because of this, we lack a sustainable, systemic approach for managing diversity (Kochan et al., 2003). Thus, from this perspective, the lack of clear analytical approaches and systems that allow for deep learning experimentation and evaluation of the management of diversity has evolved into a losing situation for all involved. This has resulted in the devaluation of individuals who are perceived 
as culturally different, reverse discrimination against members of minority groups, legal risks, demoralization, and the reinforcement of stereotypes (Bergen, Sopher, \& Foster, 2002).

On the contrary to these findings, the other perspective observes diversity within the workforce as a key success strategy that can be managed, which can result in high levels of organizational effectiveness. The extensive literature supporting the desirable benefits of diversity stresses the positive implications of diversity management and emphasizes the importance of multicultural collaboration while working on mutual projects. For instance, Cox and Blake (1991) suggest that effective diversity management could benefit organizations by increasing job satisfaction, and consequently employee retention. Research has also noted the impact on creativity that diverse employees bring to the team or organization (Cox \& Blake, 1991; McLeod \& Lobel, 1992). Watson, Kumar, and Michaelsen (1993) found that diverse groups' solutions to problems were better than those of homogeneous groups. Firms that claim to value diversity have reported an increase in recruitment and retention within their organizations (Avery \& Thomas, 2004). Therefore, today organizations are increasingly basing their HR strategic decisions on the assumption that well-planned diversity management strategies will positively impact their performance outcomes.

In order to find the key to successful diversity management, it is important to understand the essence of diversity itself. Diversity is a multifaceted and complex phenomenon, and researchers have attempted to gain a better understanding of the implications of diversity practices. Thomas and Ely (1996) define diversity as "the varied perspectives and approaches to work that members of different identity groups bring" (p. 2) while Roberson (2006) acknowledges diversity as the "differences among cultural groups as well as identity differences among group members in relation to other groups" (p. 214).

We note a key variation in these two perspectives of diversity. Roberson's definition is based on observable sources of diversity, otherwise known as surface-level diversity, while Thomas and Ely highlight the non-observable attributes of diversity, also known as deep-level diversity. Surface-level diversity includes variation in age, gender, race, etc. Deep-level diversity implies variation in perspectives and problem-solving approaches, which can influence creativity and learning, among other processes. Overall, the literature suggests that organizations do not always benefit from diversity by relying only on demographic or observable parameters in diversity management (Lorbiecki, 2001). Thomas and Ely (1996) discuss the progression of organizational diversity strategies by identifying three paradigms: the discrimination-and-fairness paradigm, the access-and-legitimacy paradigm, and the emerging learning-and-effectiveness paradigm. These have been differentiated based on the type of diversity (surface-level and deep-level) and are briefly discussed in the following section.

\section{Surface-Level Paradigms}

Two of the paradigms tend to align more with surface-level diversity. The discrimination-and-fairness paradigm is based on the principle of colorblindness (Thomas \& Ely, 1996). Colorblindness is a diversity management approach that 
ignores identity groups and treats all employees equally. This approach advocates for equal treatment of all organizational members; however, organizations operating under the discrimination-and-fairness paradigm may miss opportunities hidden in diverse employees since employees are encouraged to follow common behavioral patterns and are discouraged from utilizing their unique talents. Moreover, people who are different or do not match established rules can be viewed as problematic, even though their individuality can be the source of creativity or innovation.

The access-and-legitimacy paradigm is the other surface-level based paradigm. As opposed to the discrimination-and-fairness paradigm, this paradigm accepts and celebrates diversity. However, again diversity is viewed in terms of observable parameters such as belonging to a particular culture and ethnicity. Thus, a limitation of this perspective is the inability to fully leverage the varying perspectives that a diverse workforce can contribute. Employees are assigned to the geographic and functional areas that align with their observable factors; therefore, the organization does not effectively utilize the non-observable attributes of the employee, nor is it able to seize cross-learning opportunities.

\section{Deep Level Paradigms}

Our set of solutions to this problem will be based on the emerging perspective of viewing diversity as an opportunity for individuals from diverse backgrounds to contribute to the organization's objectives. This falls in line with the learning-andeffectiveness paradigm which Thomas and Ely (1996) purport: companies develop an outlook that enables them to incorporate employees' diverse perspectives into the main work of the organization. Since companies are pursuing foreign markets and universities are targeting more diverse students from a variety of backgrounds, organizations are particularly interested in the critical skills required to manage global projects, solve complicated multi-level problems, and interact with people from all over the world. Thus, we assert that a diverse workforce provides an incredible resource of talents, creativity, and innovation.

The learning-and-effectiveness paradigm is based on non-observable traits (deep-level diversity) such as creativity, innovation, the ability to learn, and individual thinking. Effective diversity management involves valuing and utilizing the collective talents and contributions of all individuals, regardless of differences and similarities, in ways that significantly enhance organizational performance (Thomas \& Ely, 1996). This approach aims to cultivate the positive implications of integrating differences among employees, which enables the organization to betteradjust to environmental changes. Therefore, individuals from diverse backgrounds find themselves collectively working on common problems and learning and growing due to such diversity. Emphasizing the effectiveness of the paradigm, Day and Glick (2000) mentioned that "this paradigm is different from the typical approaches to diversity based on discrimination and fairness or access and legitimacy in that the organization's members, via their differences, actually change organizations and how effectively they function because of this integration" (p.339). Thomas and Ely (1996) have identified eight preconditions for making the paradigm shift to this learning-and-effectiveness paradigm, some of which include the role of 
leadership, high standards in organizational culture, a widely understood mission, and a non-bureaucratic organizational structure.

Therefore we observe that having a diverse workforce does not automatically lead to desired outcomes unless it is managed properly. Arguably, once diversity is integrated into the organization through participant involvement, then the entire organization, not just the "diverse" individuals, can benefit from such integration. Thus, diversity can be a strategic tool for boosting performance and improving employee satisfaction.

For this research, we anchor our assertions on the learning-and-effectiveness paradigm because of its intentionality to align diversity management practices with strategic objectives and orientation (Maltbia \& Power, 2009; Pietersen, 2002). The aim of the learning-and-effectiveness paradigm of diversity management is to foster individual and organizational growth and innovation and adaption to changes in an organization's environment. This paradigm focuses on creating interventions that engage individuals, groups, organization and macro-systems through motivation, cognitive processes, and behavior (Stevens et al., 2008).

Furthermore, embedded in the learning-and-effectiveness paradigm of diversity management is the assumption that the practice of diversity management can only be advanced if organizations are willing to undergo structural and cultural transformation (Dass \& Parker, 1999; Lorbiecki, 2001). Thus, the work of diversity management encompasses both the diversifying of organizations and integrating this work with core processes. This requires organizations to develop the capacity to be inclusive multicultural organizations by moving beyond surface-level tactics that only focus on an appreciation for diversity (Stevens et al., 2008).

\section{EMERGENT SOLUTIONS BASED ON THE LEARNING-AND-EFFECTIVENESS PARADIGM}

An academic institution can be viewed as an organization in which knowledge is the "final product" that is transferred to students. However, this knowledge is a multifaceted concept that includes both explicit and tacit knowledge, development of creativity, leadership, and social experience, networking, and other valuable insights. In our education system, one of the professor's many responsibilities is to transfer such valuable knowledge. Therefore virtually any university would agree that having and valuing competent faculty that can effectively disseminate this knowledge to students is a priority. Based on the learning-and-effectiveness paradigm, universities that embody the idea of having a diverse faculty to interact with their ever-growing multicultural student body are better able to provide a more valuable "final product," that enriches both the students and the university as a whole. Establishing a diverse faculty that is motivated solely from a surface-level perspective where such diversity is driven by a fairness or function angle would not be sustainable. This enforcement would not necessarily assure that the diverse perspectives of faculty are harnessed; rather, as Thomas and Ely (1996) point out, such colorblindness practices could further propagate a groupthink culture where alternative (and potentially conflict-causing) ideas are discouraged. Moreover, this new paradigm of integrating diverse perspectives into the very nature of the university's culture will subsequently promote equal opportunity as well as value 
the cultural differences, ideals which the surface-level paradigms reflect. With the learning-and-effectiveness paradigm, diversity would result in creative ideas and innovative solutions which can only enhance the quality of education. Minority candidates have different life experiences which enable them to bring unique perspectives to both research and teaching. Students in turn will have a chance to interact with such professors who would help them to develop critical analytical thinking by exposing them to diverse perspectives.

Beyond the fact that having diverse faculty will more adequately reflect the multicultural population of university students and society at large, it has been observed that all students (not just minority students) benefit from minority representation within the faculty. A recent survey of undergraduate and graduate students at U.S. colleges and universities conducted in October 2008 revealed that minority professors have a positive impact on both minority (92\%) and non-minority students (86\%) (Bernard Hodes Group, 2008). The important survey outcomes also confirmed the significant role of the professor in the students' career-related decision making. Also of significance is the observation that $77 \%$ of students feel that they will be better prepared to work in a diverse business environment as a result of their having had a minority professor. Moreover, having a minority mentor is of noteworthy importance for underrepresented minority students since the scarcity of "natural" mentors limits the range of perspectives impacting the students. Spearheaded by an integration-minded administration, the university can create a competitive advantage by having a more diverse faculty, which in turn could be the key to attracting more diverse business students. Moreover, students will be better prepared to contribute as members of a multicultural society and will benefit from cross-ethnic social interactions after graduation (Gurin, 1999).

In spite of the intangible contributions of having a diverse faculty, there is a glaring underrepresentation of minority faculty in management education. This group represents a mere $2 \%$ of all tenured business professors. Worse yet, several of the top U.S. business schools employ no underrepresented minority faculty at all. Minorities represented $9.16 \%$ of all doctoral degrees in business conferred in 2005. Due to programs such as the $\mathrm{PhD}$ Project, the number of minority business school faculty has increased, as the program focuses on attracting minority students to business doctoral programs. According to the PhD Project, since its inception in 1994, the number of minority business school faculty has more than tripled, from 300 to over 1,000 . While this is a substantial improvement, the low minority faculty percentage still indicates a dearth. Although programs such as the $\mathrm{PhD}$ project are effective since they have significantly increased the number of URM in the business professoriate, we see a limitation of this method in that it targets successful students that are already enrolled in business-related doctoral programs. We see a great opportunity for increasing the number of potential business education doctoral students by exploring avenues for expanding the faculty recruitment pool.

\section{PHD PIPELINE ANALYSIS}

Figure 1 presents an analysis of potential educational and career pathways from the high school level to the management education professoriate level. A larger number of existing programs are currently focused on bringing talented undergraduate and/or Master's-level students into the $\mathrm{PhD}$ level. The main drawback of 
Figure 1: Analysis of pathways to the management education professoriate. This figure schematically depicts potential pathways that individuals could follow from high school all the way to the business professoriate. The circles illustrate the subsequent educational steps after high school, which noticeably decrease in size (not statistically drawn to scale) as individuals advance toward the doctoral level of education. The "Industry" rectangle on top represents an alternative career outside of the academy (usually industry-related). With one-sided arrows, we depict the student career (academic and industry) progression from high school to the professoriate, while acknowledging that students can potentially transition from the educational pipeline into industry and back. The double-sided arrows between the professoriate and industry represent the possibility for professors to transition between professoriate and industry or to simultaneously collaborate with industry organizations while maintaining an appointment within the academy.

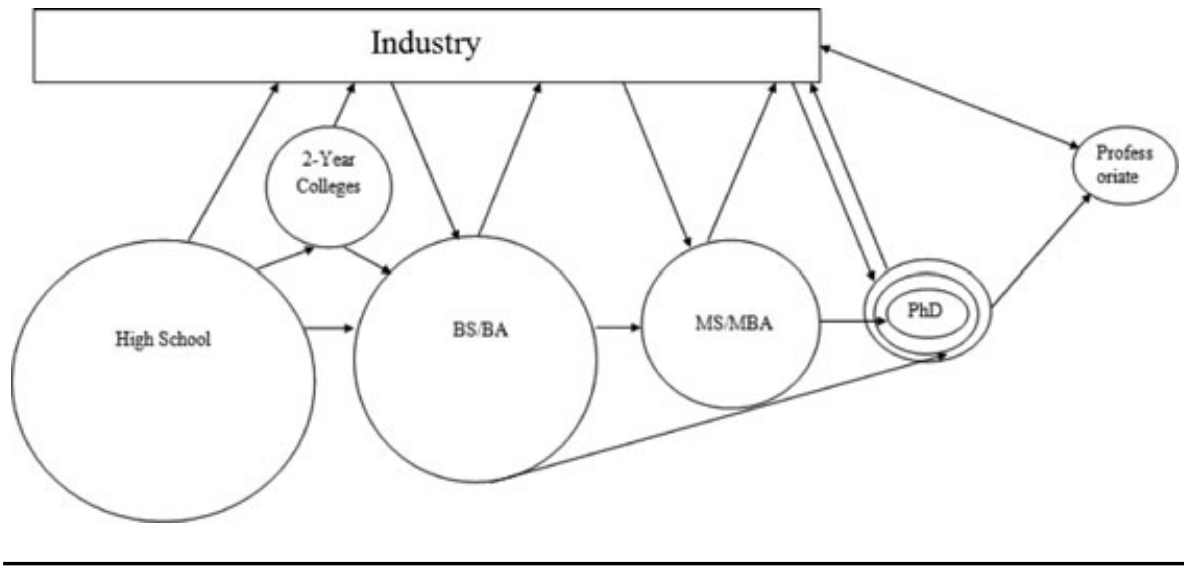

this approach is that potentially strong candidates are lost in the "larger circles" in previous steps. Therefore, we acknowledge that it is extremely important to contribute to the $\mathrm{PhD}$ pool from as early as high school for several reasons. First, since the minority population is growing at an increasing rate, the high school pool will increase and underrepresented high school graduates will form a significant percentage of potential post-secondary school entrants (U.S. Department of Education, 2007). Secondly, urban and inner city school districts, where there is a larger representation of URM students, have generally been identified as performing below standard educational level requirements (Nunez \& Cuccaro-Alamin, 1998). As we observe the fast growth in the percentage of underrepresented high school students, ongoing efforts that are being made to address this disparity must intensify.

\section{Opportunities to Increase the Pipeline}

Currently there are several existing programs that seek to expand minority representation in management education. Some support for this endeavor comes in the form of grants. For instance, the Fuqua School of Business (Duke University) recently received funding from the U.S. Department of Education to establish the PhD Pipeline Opportunity Program, which will target minority undergraduate 
students to inform and guide them toward preparing for faculty careers and will involve a national network of business faculty.

Industry grants have also funded other successful programs that seek to enhance diversity in business education. Examples of such programs include The Consortium for Graduate Study in Management and the aforementioned $\mathrm{PhD}$ Project. The goal of The Consortium (established in 1965) is to promote diversity and inclusion in American business primarily by awarding merit-based, full-tuition MBA fellowships to deserving candidates through its member universities. A number of Consortium alumni decide to go on to pursue their doctoral degrees. The PhD Project, established by The KPMG Foundation in 1994, recruits and supports URM with the goal of creating diverse business school faculties. With additional support from other corporations and partnering universities, the program targets minorities at the graduate level and provides a peer support network as they advance toward becoming business faculty.

Furthermore, there are non-minority oriented networks that focus on promoting doctoral education in business-related fields. Several universities offering doctoral degrees in business administration and economics have formed a consortium known as DocNet. DocNet seeks to educate potential students about careers in academia and focuses on increasing the pool of qualified applicants for doctoral-granting institutions by utilizing a variety of recruitment activities. The goal of expanding representation in management education is a challenge faced not only by the minority population, which is the focus of this article, but also by the general population. There is an overall shortage of business faculty with doctoral degrees, and according to the Association to advance Collegiate Schools of Business (AACSB) the number of business doctorates awarded has been declining significantly. Specifically, there was a $12 \%$ decrease in business $\mathrm{PhD}$ degrees awarded between 1995 and 2005. Furthermore, the U.S. shortage of business PhDs was predicted to have reached 2,500 by the end of the last decade. Thus, it would seem that there could be less competition for business faculty positions in the near future, which, with an expanded minority pipeline, could result in increased faculty opportunities for URM.

In the next section, we explore the problems which have been identified in the literature as having a significant influence on the recruitment, retention, and successful program completion of underrepresented minority students at all educational steps. In discussing these problems, we also observe opportunities for enlarging the pipeline at the different stages.

\section{KEY ISSUES TO ADDRESS}

\section{Educational Experience}

We have observed that the educational experience of underrepresented students contributes to the likelihood that these students will not only be successful in their current stage, but also are more likely to progress to subsequent stages. Leading up to the high school level, it is important to note that the retention level among URM students is already observably lower than their non-URM counterparts (Varhkey \& Applewhite-Lozano, 1985). There are numerous reasons for this outcome. One 
is the level of responsibility that many URM students have at this stage, which includes other obligations, such as part-time jobs or having to serve as caretakers when parents/guardians are not present. Fewer non-URM students are faced with similar responsibilities, which could suggest a connection to historical inequities. Nevertheless, such obligations often do not allow URM students to dedicate the time necessary for preparing for post-secondary opportunities. Consequently, such students are more likely to achieve lower GPAs, thus making it more challenging to gain admission and compete at the college level (Mingle, 1987).

We propose addressing the problem from the high school level, given that the high school graduation rate for URM is lower than their non-URM students counterparts. There is a noticeable domino-effect outcome in considering the lack of preparedness of URM students at the collegiate level. For instance, even after they have been accepted into college, the lack of role models and mentoring support available to URM students in college can certainly influence the success outcome of such students and their decision to pursue higher/graduate levels of study. Specifically, this lack of networking and mentoring support can result in less-successful $\mathrm{PhD}$ application packages and less exposure to graduate education opportunities and beyond. Moreover, it is important to acknowledge that the aforementioned educational experiences of URM students and the subsequent implications do not necessarily suggest that these students are any less capable of being successful at all levels of higher education (and the professoriate) than their non-URM counterparts. Thus, this solutions-oriented article joins the ongoing discussion in exploring and highlighting practices that can address the existing minority underrepresentation in management education.

\section{Motivation}

We also consider motivation to be an important factor in enabling URM students to successfully transition through the pipeline process. Motivation throughout the pipeline is relevant to this study from two dimensions. The first relates to the motivation and determination associated with completing the respective degree. A significant number of URM students today are first-generation college students from lower-income families (US Department of Education, 2005). "The results show that such students are at a distinct disadvantage in gaining access to postsecondary education. Even those who overcome the barriers and do enroll have difficulty remaining enrolled and attaining a degree" (Horn, Nunez, \& Bobbitt, 2000, p. 1). First generation students face a more challenging progression from high school to postsecondary education than their peers because of numerous factors, including anxieties, dislocations, and social and academic transitions (Pascarella, Pierson, Wolniak, \& Terenzini, 2004). Being a first generation student also influences retention negatively. For instance, research indicates that the first-generation students are "more likely to leave a four-year institution at the end of the first year, less likely to remain enrolled in a four-year institution, and are less likely to stay enrolled or attain a bachelor's degree after five years" (Pascarella et al., 2004, p. 250). Furthermore, research also suggests that a first generation student is more likely to have a more challenging and complicated educational experience (than non-first generation students) due to the lack of parental support and role models 
in the family (Freeman, 1997). Thus, we conclude that first-generation students (and consequently a significant percentage of URM students) could experience motivational challenges in progressing through the pipeline.

Another important factor affecting motivation is that URM students have an observable desire to start serving within their community as soon as possible (Hammond, 1995). Family responsibilities and the need to give back to ethnic communities are important cultural issues affecting minorities (Elliott, 1994). Having fewer examples of family members in the professoriate, URM students are potentially more likely to choose "less-challenging" careers as more realistic opportunities to give back to the community (Payton, White, \& Mbarika, 2005).

The second motivational dimension is related to the desire to pursue a career in management education. Additional motivational challenges arise while working toward completing the $\mathrm{PhD}$ degree as individuals identify some opportunity costs associated with obtaining the doctoral degree in business-related areas. People assume that although pursuing an academic career could be a desirable opportunity, it involves some considerable sacrifices: long-term commitment, lower income (lower compensation levels than in industry), and uncertainty in finding attractive employment opportunities afterward. Seeking a doctoral degree entails committing to another four to six years of school while contemplating the aforementioned factors. Therefore, there is a high cost of risks while industry positions appear to offer more immediate financial rewards, higher income, and greater opportunities for success. However, a PhD Project participant who entered a doctoral program after working in industry for some years said that "the idea of [the] biggest commission doesn't excite him anymore...[he] would rather be helping to teach the next generation of professionals and researchers" (Mangan, 2006, p. 2). As with the previous section on educational experiences of URM students, these motivational factors do not imply that URM students are "less able" than their non-URM counterparts. Rather, these factors highlight the resulting outcomes that transitional challenges and a lack of awareness (discussed further in the next section) could have, which can be de-motivating to students of any ethnic background.

\section{Awareness}

The lack of awareness of a career opportunity in business/management academia plays a crucial role since it is an obvious precondition for considering business academia as a career option. There are several factors related to this unawareness. For instance, the AACSB identified some myths about pursuing doctoral study resulting in a career in business academia (AACSB, 2003). Such myths include the belief that doctoral studies are self-funded, faculty pay is low compared to a traditional business career, and candidates must have earned an MBA or other Master's degree to apply for a doctoral program in business. Moreover, some of the students that do go into business education do not really view teaching as a career path and are not in a position to appreciate the intellectual and even economic strengths of this path. The desire to become a business professor cannot originate without sufficient information and vivid examples. Previously-mentioned URMrelated influences including being a first-generation college-attendee and the dearth 
of minority representatives in management education that could serve as mentors are both factors that could undoubtedly influence such awareness.

\section{Social Environment}

The social environment refers to how comfortable URM students and professors feel on campus, among classmates, and colleagues. Payton and Jackson (1999) note that one of the main reasons minority students are underrepresented is a result of social isolation that stems from campus climates that can be inhospitable as well as an overall "non-pluralistic institutional orientation." Arner and Yates (1979) observe that the institutional atmosphere is the most important factor for the successful recruitment and retention of minority students.

Research suggests that displays of "white supremacy" still occur; for instance, Thomas and Wetlaufe (1997) talk about existing assumptions that URM students are not competent or not smart enough and that these individuals are expected to fail. These assumptions can lead to the aforementioned reduced motivation that professors have regarding working with URM students. Teresa Hammond (1995) also mentions institutional racism as a social barrier that influences the interaction between doctoral students and faculty, low expectations from professors, mistrust from students, and resentment from other doctoral students. Table 1 highlights a summary of the problems (and reflects opportunities for improvement) that impede progression through the different stages of the pipeline, from high school to the $\mathrm{PhD}$ level.

\section{SOLUTIONS}

The previous section discussed key problems that influence the progression of URM students through the pipeline. In this section, we propose strategic solutions to address these problems. Table 1 highlights these solutions as they pertain to the problems that were previously discussed. Please see the Appendix (Figure 2) for a diagram that summarizes our proposed solutions. We differentiate our solutions based on the following criteria:

1. Our proposed solutions are supported by the Thomas and Ely learningand-effectiveness paradigm. In the literature review section, we addressed the significance of integrating diversity within the organization through participant involvement. In doing so, both URM and non-URM students benefit from the implementation of such integration.

2. We propose to start tracking the pool at the high school level. Most first generation students are more likely to delay postsecondary entry, begin at a two-year institution (big leak from the pipeline), and attend part time and discontinuously (First-Generation Students in Postsecondary Education). African Americans and Hispanics are overrepresented in two year institutions; their enrollment is sporadic and extended and their persistence rates and transfer rates to four year degree-granting institutions are disturbingly low (McConnell, 1993). Elliott (1994) acknowledged that URM students after high school graduation are "raw" since students have not been prepared for post-secondary education or advanced careers; 
Table 1: Proposed actions to increase the number of minority students within the pipeline. The table offers the set of solutions (placed in the left-most column) which can be effectively addressed across a sequence of educational steps (placed horizontally across the top row) that support the student along the path from high school to the professoriate level. The set of solutions are specific actions that correspond to the key issues discussed in the previous section. If the solution is considered appropriate at a particular educational step, the cell in the intersection is marked with an $\mathrm{X}$.

\begin{tabular}{lccccc}
\hline & High school & BS & MS/MBA & PhD & Professoriate \\
\hline $\begin{array}{l}\text { Educational experience } \\
\quad \text { Mentorship }\end{array}$ & $\mathrm{X}$ & $\mathrm{X}$ & $\mathrm{X}$ & $\mathrm{X}$ & \\
$\quad \begin{array}{l}\text { PhD preparedness (Info sessions; } \\
\quad \text { Admission issues) }\end{array}$ & & $\mathrm{X}$ & $\mathrm{X}$ & & \\
$\begin{array}{l}\text { Motivation } \\
\text { Mentorship }\end{array}$ & $\mathrm{X}$ & $\mathrm{X}$ & $\mathrm{X}$ & $\mathrm{X}$ & \\
$\quad$ Networks & $\mathrm{X}$ & $\mathrm{X}$ & $\mathrm{X}$ & $\mathrm{X}$ & $\mathrm{X}$ \\
$\quad$ Sponsored speakers & $\mathrm{X}$ & $\mathrm{X}$ & $\mathrm{X}$ & $\mathrm{X}$ & \\
$\quad$ Fellowship programs & $\mathrm{X}$ & $\mathrm{X}$ & $\mathrm{X}$ & $\mathrm{X}$ & \\
$\quad$ Opportunity to be a mentor for other & $\mathrm{X}$ & & & $\mathrm{X}$ & \\
$\quad$ students & & & & & \\
Awareness & $\mathrm{X}$ & $\mathrm{X}$ & $\mathrm{X}$ & $\mathrm{X}$ & $\mathrm{X}$ \\
Social environment & $\mathrm{X}$ & $\mathrm{X}$ & $\mathrm{X}$ & $\mathrm{X}$ & $\mathrm{X}$ \\
$\quad$ Organization culture & & & & & \\
$\quad$ Diversity within top management & $\mathrm{X}$ & $\mathrm{X}$ & $\mathrm{X}$ & $\mathrm{X}$ & $\mathrm{X}$ \\
$\quad$ top bottom approach) & & & & & \\
$\quad$ Social inclusion & & & & & \\
\hline
\end{tabular}

the necessity to "catch" students in high school and junior high is also emphasized. Therefore, while other programs (e.g., The Consortium, The PhD Project) offer support at the graduate level, we suggest the broad approach of increasing the initial pool and minimizing the attrition rate as students progress through the pipeline process. Therefore, more students are aware and are fed into established and successful programs such as The PhD Project.

3. With regard to implementation, the solution set should be adjusted for each individual academic institution since each university varies in terms of available resources (human and financial). It is also worth noting that our proposed solutions are complementary and interdependent.

\section{Solution 1: Improving the Educational Experience}

Numerous studies have identified the significant role of mentors in recruiting and retention by improving the protégé's performance and satisfaction (Bursch, 1985; Green \& Bauer, 1995; Kram \& Isabella, 1985). Levinson, Darrow, Klein, Levinson, and McKee (1978) note that mentoring is a relationship that can be both "complex and developmentally important"; thus, establishing a mentoring relationship at an early stage, such as the high school level, can be beneficial to the sustained success 
of URM students. It is important to acknowledge that mentoring relationships with "surface-level" diverse participants can be equally as successful as relationships with "surface-level" similar participants.

There is a plethora of anecdotal insights about the significance of URM students having URM mentors and role models that can inspire, motivate, and mentor them to pursue careers as business school professors. Social support from a URM role model is important because it enables URM students to envision how they can pursue a particular career (Freeman, 1997; Jepson \& Peri, 2002). Also, as a byproduct of interacting with URM role models, URM students develop the mental cognition needed to prepare for graduate school and the capacity to remove obstacles and find solutions to address the opportunity cost of attending graduate school (Stewart, Williamson, \& King, 2008).

With regard to "deep-level" mentoring relationships, Olian, Stephen, Giannantonio, and Feren (1988) found that a key attraction factor for the protégé is a mentor's interpersonal competence followed by the manager's integration into a network. Both of these are "deep-level" or non-observable factors. Observable or surface-level factors such as gender and age did not affect the protégés' attraction. This suggests that attempts should be made to match the students' career interest, majors, and hobbies with appropriate interests and backgrounds of the mentors. It is worth noting that a mentoring relationship can be initiated by the mentor as well as the protégé.

The pipeline process can provide an effective mentoring strategy in that as students progress through the pipeline, they can serve as mentors to students in previous stages. This can be done informally or formally, through student organizations. Mentoring can involve high school visitations, answering questions, increasing awareness, and being involved in online social networks.

Professors can also be motivated to formally or informally mentor URM students. However, as suggested by the learning-and-effectiveness paradigm, sustained outcomes are achieved when URM students are involved in research that covers a wide range of topics based on the students' interests, and are not only engaged in research that is tied to URM-related topics. As was emphasized earlier, the most effective mentors may not necessarily be of the same "surface-level" diversity background.

In addition to mentoring, several other factors can improve the educational experience at all stages of the pipeline. Such factors include participating in programs dedicated to preparing students for doctoral studies, such as the McNair Scholars Program. Many institutions have academic offices that provide academic skills workshops and information sessions that inform and better-prepare students to progress through the pipeline.

\section{Solution 2: Addressing Motivation}

We evaluated motivation from two perspectives as it relates to the focus of this article: motivating students to graduate at the various stages of the pipeline and motivating students to consider an academic career in management education. Research on motivation suggests that intrinsic motivation is more effective (than extrinsic) at achieving desirable performance (Vansteenkiste \& Lens, 2006). Thus 
presenting the necessary tools and information to students such that they truly take ownership of the fact that they can be successful and that management education is a viable option. Such tools/information includes the aforementioned mentoring, networking, and fellowship programs, among other strategies.

\section{Solution 3: Addressing Awareness}

The current shortage of business school academicians has been steadily worsening in recent years. A key reason for this shortage is a lack of awareness about the opportunities associated with an academic career in management education, not just among URM students, but with all students. Thus, while institutions could be more proactive in recruiting ideal candidates, students (especially at the high school level) can also be more exposed to the idea of such a career by highlighting the rewards associated with teaching and by emphasizing the fact that an academic can also serve on corporate boards and be a business consultant.

In addition, the myths related to pursing doctoral studies in a business field should also be addressed at early education stages. While a significant number of students believe that they will have to go into debt to pursue a doctoral degree, a vast majority of new doctoral students are funded by university or outside fellowships. According to the National Science Foundation, a majority of doctoral graduates had less than $\$ 10,000$ in debt from their doctoral studies. In addition, students tend to believe that faculty pay is lower compared to a traditional business career. Specifically, an AACSB study indicated that students underestimated compensation by at least 39\% of the true level of earnings for business PhD's in academia. Yet another misconception is that doctoral candidates must have also earned a Master's or MBA degree. However, individuals with an undergraduate degree are also eligible to apply for $\mathrm{PhD}$ programs. Consequently some candidates also end up receiving their Master's degree along the way. Thus, providing exposure to such information through mentoring, pipeline programs, and academic and counseling offices could potentially open more pathways toward careers in management education.

\section{Solution 4: Improving the Social Environment}

The management literature emphasizes the role of senior executives in driving the culture of an organization as well as enacting change (Kilduff, Angelmar, \& Mehra, 2000). Therefore, the significant role of the academic administration in establishing an environment that perpetuates the learning-and-effectiveness paradigm cannot be overstated. This begins with having a shared vision toward this mission of diversity. There needs to be a philosophical commitment to the belief that diversity enriches the total university community. The mission must be designed to permeate the entire university with an atmosphere that suggests that all students can expect a sense of community while they pursue their educational goals. This concept must be supported at all levels of administration (Varhkey \& Applewhite-Lozano, 1985). Specifically, administration and faculty members should be aware of projects, programs, and goals that are aimed at reaching university objectives.

It is also important that universities employ a top-down approach, meaning that they should ensure that top-level administrators share in the same 
diversity-driven mission and have a uniform sense of direction. Universities should be diligent about attracting resources (open-minded individuals, support for program development and implementation) that align with promoting deeplevel diversity. As the learning-and-effectiveness paradigm highlights, it is the deep-level diversity that leads to a more robust and more sustained implementation of university initiatives that target underrepresentation in management education.

Social inclusion is an important aspect of creating a social environment that enables success among URM students. Retention is positively correlated with a high degree of student involvement. Social inclusion helps to prevent the distance that might exist between URM and non-URM colleagues which can appear due to common assumptions. Academic institutions should strive to create an environment of inclusion and involvement as this would lead to improved relationships between and among both students and faculty.

\section{LIMITATIONS AND DIRECTIONS FOR FUTURE RESEARCH}

The conceptual nature of this article is a principal limitation. Since the goal of this research was to develop an innovative approach to address the issue of minority underrepresentation within the business faculty, the solution we offer is a complex set of strategies which primarily targets students at the high school level. Considering the complexity of the approach, there are challenges for conducting empirical research that would corroborate the solutions. First of all, the implementation would necessitate a tremendous amount of resources since it requires significant investments at each educational step. For instance, a specific goal could involve developing infrastructures which would provide a platform for educational bridge programs starting at the high school level. Educational bridge programs reach high school students through summer activities and extra-curricular activities during the school year. These programs prime the pipeline by providing a roadmap that prepares students for college. Students are also exposed to positive role models that can mentor students and discuss potential career paths at this early stage of the pipeline (Jepson \& Perl, 2002).

Secondly, this is a long-term approach that will produce noteworthy results only after an extensive period of time as the time span between high school completion and entering the management professoriate could range from approximately 8 to 12 years. Thirdly, in conducting the background research for this study, we explored multiple sources of information to find statistics about students exiting and re-entering the educational pipeline; however, we were challenged by the lack of sufficient data. Nevertheless, this information can be difficult to track since students may leave and return to the pipeline several times, shift to different education or vocational fields, or change their career plans during their educational journey. Thus, there exists the challenge of having to track and measure results, a drawback that similar longitudinal approaches have faced. Finally, the article focused on internal solutions which can be implemented and controlled by the academic institution. However, there is a great potential for future research on a set of solutions that can be built around "external" (not solely controllable by academic institutions) forces, such as government, media, online social networks, 
and educational organizations such as AASCB and NCES (National Center for Education Statistics).

We would like to emphasize the importance of future research regarding the role of online social networks in increasing the URM representation within the business professoriate. Recent research revealed a strong trend of the extensive usage of social networks such as Facebook, Twitter, blogs, and forums by URM (Smith, 2010). Moreover, not only are they more active in online communication, but they also view social Web tools differently and tend to utilize them beyond only entertainment purposes. For instance, Web tools are regarded as an important source of information and provide a platform to discuss significant issues and events. During our research we explored the effectiveness of existing online solutions such as MyPhDNetwork, an online social network created exclusively for The PhD Project Doctoral Student Association (DSA) members. We consider social networks to be a catalyst for disseminating information and increasing awareness about academic opportunities as well as promoting the academic career path.

The impact of the media is another focus for further research. Today, the images of people of color that the media bring to the larger audience influences the limited perceptions affiliated with viewing URM as business professors. It negatively influences URM individuals who are making important decisions about their career paths as well as the non-URM population, who forms distorted opinions about the success of URM professors in the management education field. Moreover, in addition to online social networks, the media can be a great resource for educating URM about career opportunities in academia. By combining these two powerfully influential forces, the issue of lack of awareness and motivation can be addressed.

Industry and government are currently helping to address the problem of increasing the underrepresentation among business faculty by financing existing programs and offering scholarships and fellowships at different stages of the pipeline. However, considering the complexity of our proposed solutions, their roles can be shifted to levels that could expand beyond financial support. Therefore, a new look at the role of government and industry can also be a potential topic for a future research.

\section{CONCLUSION}

In closing, we propose that creating a diverse learning environment in management education calls for developing a minority pipeline of faculty. To support this assertion, we draw upon theoretical frameworks from the diversity management literature. In particular, we advocate the learning-and-effectiveness paradigm. This approach involves learning how to embrace diversity by integrating differences to cultivate capabilities and adapt environmental conditions. The learning-andeffectiveness approach demands system thinking to solve organizational problems relating to diversity and culture that are inclusive of differences. In the context of developing a faculty pipeline for management education, we contend that this starts at the high school level, and at each subsequent phase of the educational 
journey there are opportunities to develop this pipeline. The work of developing a pipeline is so complex a task that we propose a three-prong solution.

This three-prong solution is grounded in the appropriate education experiences that one needs to become a faculty member. It takes into account the factors that motivate individuals to pursue a career in academia. At the core of this motivation is creating high-quality connections through mentors, networks, fellowship programs, and workshops that exposed minorities to careers in academia, and more specifically business higher education with the goal of building an awareness of doctoral studies. Just as important as awareness is the need for business schools to create an inclusive social environment. This requires moving beyond the minimization of differences and capitalizing on the richness that diversity has to offer in the classroom, to administrative work, and in the research arena. Only when business schools develop an infrastructure that respects and supports diversity will they be in the position to adapt to a multicultural environment and reap the benefits of diversity.

\section{REFERENCES}

AACSB International. (2003). Sustaining Scholarship in Business Schools, http://www.gfme.org/issues/pdfs/SustainingScholarship.pdf.

AACSB International. (2010). Business School Data Trends and 2010 List of Accredited Schools, http://www.aacsb.edu/publications/businesseducation/ 2010-Data-Trends.pdf.

Arner, R., \& Yates, A. (1979). Recruitment of minority graduate students. Communicator, (9), 1-5.

Avery, D., \& Thomas, K. M. (2004). Blending content and contact: The roles of diversity curriculum and campus heterogeneity in fostering diversity management competency. Academy of Management Learning and Education, 3(4), 380-396.

Barak, M. (2005). Managing Diversity: Toward a Globally Inclusive Society. Thousand Oaks, CA: Sage.

Bell, M. P., Connerley, M. L., \& Cocchiara, F. K. (2009). The case for mandatory diversity education. Academy of Management and Learning Education, 8(4), 597-609.

Bergen, C., Barlow, S., \& Foster, T. (2002). Unintended negative effects of diversity management. Public Personnel Management, 31(2), 239-251.

Bernard Hodes Group. (2008). PhD project student survey report, http://www. phdproject.com/downloads/Students_Report_6-9-08.pdf.

Busch J. W. (1985). Mentoring in graduate schools of education: Mentors' perceptions. American Educational Research Journal, 22(2), 257-265.

Cox, T., \& Blake, S. (1991). Managing cultural diversity: Implications for organizational competitiveness. The Academy of Management Executive, 5(3), 45-56. 
Daniels, C. (2001). Too diverse for our own good? Fortune, 144(1), 116.

Dass, P., \& Parker, B. (1999). Strategies for managing human resource diversity: From resistance to learning. The Academy of Management Executive, 13(2), $68-80$.

Day, N., \& Glick, B. J. (2000). Teaching diversity: A study of organizational needs and diversity curriculum in higher education. Journal of Management Education, 24(3), 338-352.

Elliott, J. A. (1994). Enhancing diversity in business doctoral education. Selections, 11(1), 8-11.

Freeman, K. (1997). African American's participation in higher education: African American high-school students' perspectives. The Journal of Higher Education, 68(5), 523-550.

Freidman, T. (2005). The World is Flat. New York: Farras, Straus \& Giroux.

Gagnon, R.J., Totten, J. W., \& Morgan, S. (2007). An exploratory analysis of minority business cases: A follow-up. Journal of the Academy of Business Education, 8 (Proceedings), http://www.abe.sju.edu/proc2007/gagnon.pdf [accessed January 13, 2011]

Green, S. G., \& Bauer, T. N. (1995). Supervisory mentoring by advisers: Relationship with doctoral student potential, productivity, and commitment. Personnel Psychology, 48(3), 537-562.

Grubb, H. J., \& Ollendick, T. H. (1986). The culture distance perspective: An exploratory analysis of its effect on learning and intelligence. International Journal of Intercultural Relations, 10(4), 399-414.

Gurin, P. (1999). New research on the benefits of diversity in college and beyond: An empirical analysis., http://www.diversityweb.org/Digest/Sp99/ benefits.html. [accessed March 1, 2011]

Hammond, T. D. (1995). Some considerations in attracting and retaining AfricanAmerican doctoral candidates in accounting. Issues in Accounting Education, 10(1), 143-158.

Harvey, B. H. (1999). Technology, diversity and work culture-key trends in the next millennium. HR Magazine, 44(11), 58-59.

Horn, L., Nunez, A., \& Bobbitt, L. (2000). Mapping the road to college: First-generation students' math track, planning strategies, and context of support. National Center for Education Statistics, http://nces.ed.gov/ pubs2000/2000153.pdf

Jepson, A., \& Peri, T. (2002). Priming the Pipeline. SIGCSE Bulletin, 34(2), 36-39.

Kilduff, M., Angelmar, R., \& Mehra, A. (2000). Top management-team diversity and firm performance: Examining the role of cognitions. Organization Science, 11(1), 21-34.

Kochan, T., Bezrukova, K., Ely, R., Jackson, S., Joshi, A., Jehn, K., Leonard, J., Levine, D., \& Thomas, D. (2003). The effects of diversity on business 
performance: Report of the diversity research network. Human Resource Management, 42(1), 3-21.

Kram K. E., \& Isabella L. A. (1985). Mentorship alternatives: The role of peer relationships in career development. Academy of Management Journal, 28(1), 110-132.

Kuczynski, S. (1999). If diversity, then higher profits? HR Magazine, 44(13), 66-74.

Lee, S. H., Shenkar, O., \& Li, J. (2008). Cultural distance, investment flow, and control in cross-border cooperation. Strategic Management Journal, 29(10), $1117-1125$.

Lorbiecki, A. (2001). Changing views on diversity management: The rise of the learning perspective and the need to recognize social and political contradictions. Management Learning, 32(3), 345-361.

Levinson, D. J., Darrow, C. N., Klein, E. B., Levinson, M. A., \& McKee, B. (1978). Season of a main life. New York: Knopf.

Maltbia, T., \& Power, A. (2009). A Leader's Guide To Leveraging Diversity. Burlington, MA: Butterworth-Heinemann.

Mangan, K. S. (2006). Career track to tenure track. The Chronicle of Higher Education, 53(1), http://www.phdproject.org/downloads/Chronicle_ tenuretrack.pdf.

Maruyama, G., Moreno, J., Gudeman, R., \& Marin, P. (2000). Does Diversity Make a Difference? Three Research Studies on Diversity in College Classrooms. Washington, DC: American Council on Education.

Mingle, J. (1987). Focus on minorities: Trends in higher education preparation and success. Denver: Education Commission of the Higher Education Executive Offices.

McConnell, E. C. (1993). Minority student attrition research: Higher education's challenge for human resource development. Educational Researcher, 22(7), 24-30.

McLeod, P. L., \& Lobel, S. A. (1992). The effects of ethnic diversity on idea generation in small groups. Academy of Management Best Paper Proceedings, 227-231.

Nunez, A., \& Cuccaro-Alamin, S. (1998). First-Generation Students: Undergraduates Whose Parents Never Enrolled in Postsecondary Education (NCES 98-082). Washington, DC: National Center for Education Statistics, U.S. Government Printing Office.

Olian, J. D., Stephen, C. J., Giannantonio, C. M., \& Feren, D. B. (1988). What do protégés look for in a Mentor? Results of three experimental studies. Journal of Vocational Behavior, 33(1), 15-37.

Pascarella, E. T., Pierson, C. T., Wolniak, G. C., \& Terenzini, P.T. (2004). Firstgeneration college students: Additional evidence on college experiences. The Journal of Higher Education, 75(3), 249-284. 
Payton, F. C., \& Jackson, C. (1999). Ethnic diversity in IS: What are current Ph.D. students saying? ACM SIGCPR Computer Personnel, 20(3), 2739.

Payton, F. C., White, S. D., \& Mbarika, V. (2005). A re-examination of racioethnic imbalance of IS doctorates: Changing the face of the IS classroom. Journal of the Association for Information Systems, 6(1), 37-51.

Pietersen, W. (2002). Reinventing Strategy: Using Strategic Learning to Create and Sustain Breakthrough Performance. New York: Wiley.

Roberson, Q. (2006). Disentangling the meaning of diversity and inclusion in organizations. Group and Organization Management, 31(2), 212236.

Smith, A. (2010). Technology trends among people of color. http://www. pewinternet.org/.

Stahl, G. K., Mäkelä, K., Zander, L., \& Maznevski, M. L. (2010). A look at the bright side of multicultural team diversity. Scandinavian Journal of Management, 26(4), 439-447.

Stevens, F., Plaut, V., \& Sanchez-Burks, J. (2008). Unlocking the benefits of diversity: All inclusive multiculturalism and positive organizational change. Journal of Applied Behavioral Science, 44(1), 116-133.

Stewart, M., Williamson, I., \& King, J. (2008). Who wants to be a Business PhD? Exploring minority entry into the faculty pipeline. Academy of Management Learning \& Education, 7(1), 42-55.

Thomas, D. A., \& Ely, R. J. (1996). Making differences matter: A new paradigm for managing diversity. Harvard Business Review, 74(5), 79-90.

Thomas, D. A., \& Wetlaufe, S. (1997). A question of color: A debate on race in the US workplace. Harvard Business Review, 75(5), 118-132.

U.S. Census Bureau. (2009). http://www.census.gov/.

U.S. Department of Education. National Center for Education Statistic. (2005). First-Generation Students in Postsecondary Education, http://nces.ed.gov/pubs2005/2005171.pdf

U.S. Department of Education. National Center for Education Statistic. (2007). Status and Trends in the Education of Racial and Ethnic Minorities, http://nces.ed.gov/pubs2007/minoritytrends/

Vansteenkiste, M., \& Lens, W. (2006). Intrinsic versus extrinsic goal contents in self-determination theory: Another look at the quality of academic motivation. Educational Psychologist, 41(1), 19-31.

Varhkey, S., \& Applewhite-Lozano, S. (1985). A Recruitment and retention plan for students from minority groups. Journal of college student personnel, 26, $77-78$.

Watson, W. E., Kumar, K., \& Michaelsen, L. K. (1993). Cultural diversity's impact on interaction process and performance: Comparing homogeneous and diverse tasks groups. Academy of Management Journal, 36(3), 590602. 


\section{APPENDIX}

Figure 2: Proposed solutions categorized by identified problems.

Solutions should be based on:

- Applying the learning-and-effectiveness paradigm

Starting at the high school stage of the pipeline

Adjusting the set of solutions for each institution

\section{SOLUTIONS}

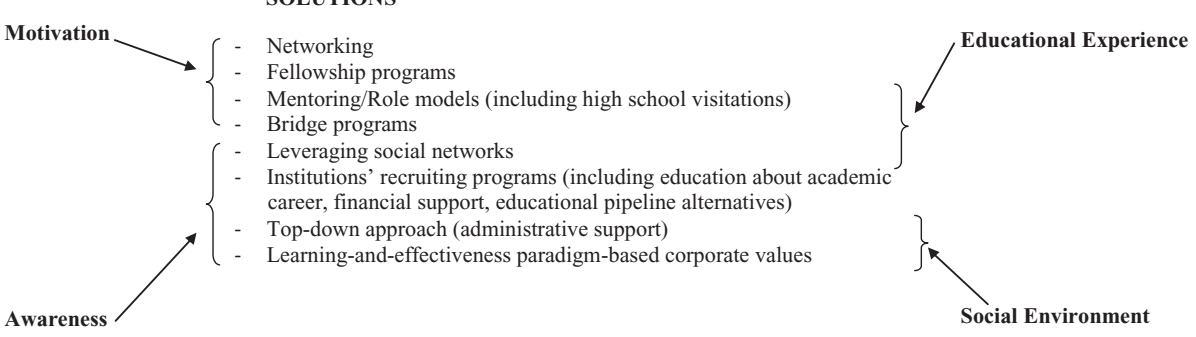

Joy Oguntebi is an assistant professor of management at the Rochester Institute of Technology's Saunders College of Business. She received her PhD from the University of Michigan in Industrial \& Operations Engineering, with a concentration in engineering management. She has conducted research that explores the effectiveness of knowledge management and collaborative mechanisms within geographically dispersed team settings. Her research interests include organizational learning, knowledge management, team effectiveness, global teams, and cultural intelligence and she has presented her research at several domestic and international conferences. Dr. Oguntebi teaches courses in Management and International Business.

Maria Shcherbakova has an MBA degree in Marketing and International Business from Rochester Institute of Technology. Her bachelor degree in Computer Science was received in Kazan State University, Kazan, Russia.

Lynn P. Wooten joined University of Michigan Business School faculty in 1998 and earned her PhD from the Ross School of Business at the University of Michigan. She teaches organizational behavior, nonprofit management, and strategic consulting courses. She is currently the co-director of the Center for Positive Organizational Scholarship at the Ross School of Business. Her current research focuses on how changes in workforce demographics and the knowledge economy influence the implementation of strategic human resource management practices. In particular, she examines how these human resource management practices affect the performance of organizations and employee-related outcomes. 